\title{
Development of an ES2-type point machine (monitoring of point machine)
}

\author{
N. Obata ${ }^{1}$, T. Ichikura ${ }^{1}$, H. Narita ${ }^{2}$ \& H. Tanaka ${ }^{3}$ \\ ${ }^{I}$ Technical Center, \\ The Research and Development Center of JR East Group, Japan \\ ${ }^{2}$ Quality Control Department, Railway Signal Division, \\ Kyosan Electric Mfg. Co., Ltd., Japan \\ ${ }^{3}$ Signal Systems Third Designing Department, Railway Signal Division, \\ Kyosan Electric Mfg. Co., Ltd., Japan
}

\begin{abstract}
The existing monitoring system for switch-point machines was introduced in order to inform dispatchers and maintenance staff when there is a breakdown or a decrease in the functioning of signal equipment. Past monitoring has been limited to the locking error detector. When the ES-type point machine with a servo-motor was introduced in 2002, it became possible to monitor other information, such as voltage, switching time, stroke, and torque. The next step was development of the ES2-type point machine, an improved ES type with greater stability.

In the future, trouble with the turnout mechanism will also be monitored through techniques now under research. We will also research how to find signs of trouble before there is a breakdown.
\end{abstract}

Keywords: point machine, monitoring, maintenance.

\section{Introduction}

Recently the number of signal devices has been increasing. When signal equipment breaks down, this has a major impact on railway transportation. The existing monitoring system was introduced in order to inform dispatchers and maintenance staff if the functioning of signal equipment has decreased or there is a breakdown. In Japan, point machines have an indirect lock system. Monitoring of the point machine in the past has been directed toward locking errors. In the 
ES-type point machine, which recently was developed, the functions that can be monitored include not only the lock, but also the torque, stroke, voltage and other factors. The ES2-type point machine was developed by improving the ES-type point machine to increase its reliability.

In this paper, the function of monitoring by the ES-type point machine is described, and the capabilities that are improved with the ES2-type point machine are also described.

\section{Summary of the existing monitoring system}

The existing monitoring system was introduced so that dispatchers and maintenance staff would be aware of any breakdowns or decrease in function of signal equipment. It consists of:

(1) Sensor devices that measure the operating condition of the equipment and various other data

(2) A control and display unit that indicates and accumulates the information from each sensor

(3) Transmission devices that transmit information between the sensor devices and the control display.

The system requires communication from the control and display unit to the sensor devices, and the answers from the sensor devices to requests are returned to the control and display unit. The transmission device provides this telecommunication link.

An example of the display control is shown in figure 2. Each time that a breakdown occurs or recovery is made, the system indicates and records date and time, location, sensor name, object equipment, type of breakdown, and whether

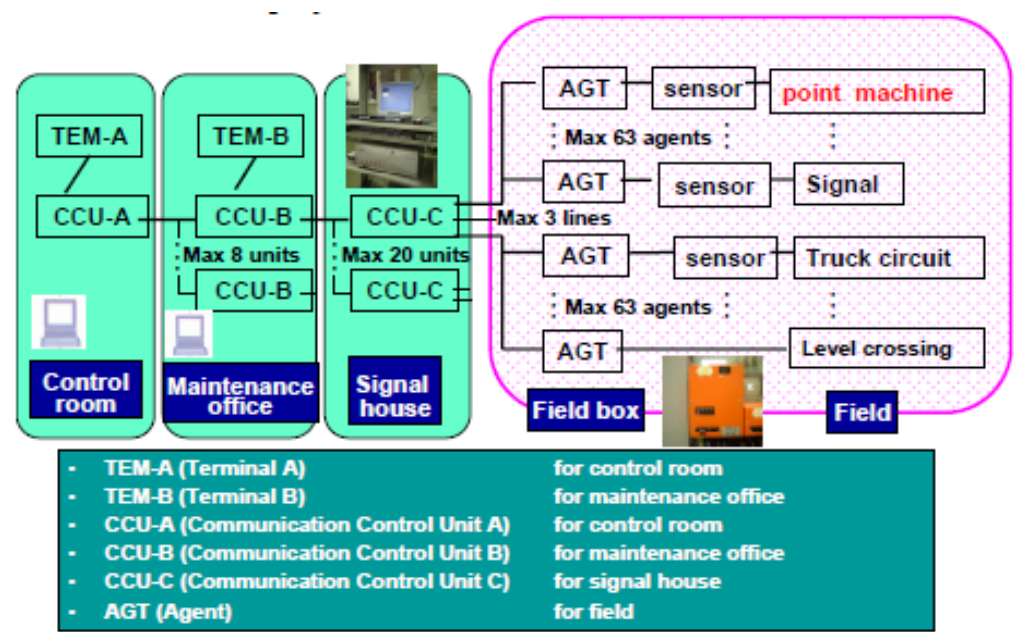

Figure 1: $\quad$ System structure of the existing monitoring system. 


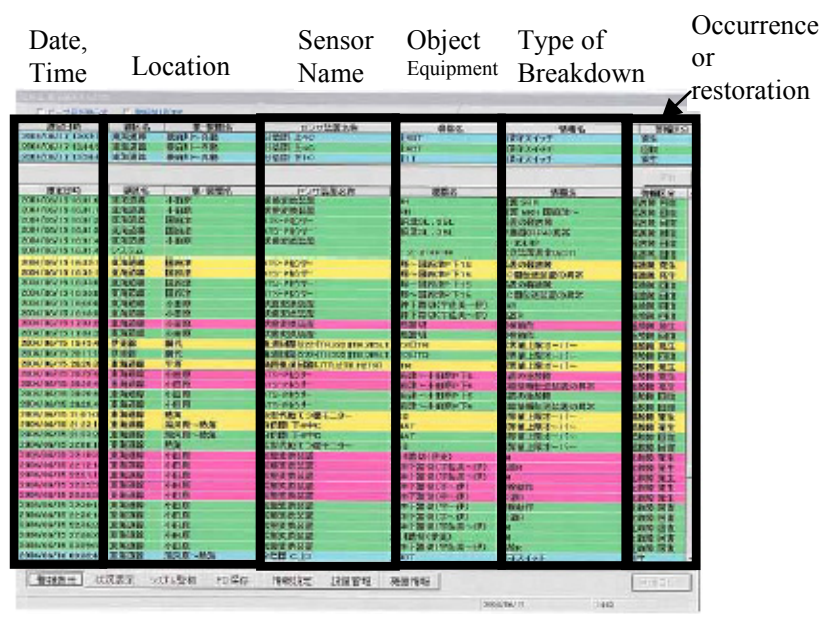

Figure 2: $\quad$ Example of the display control.

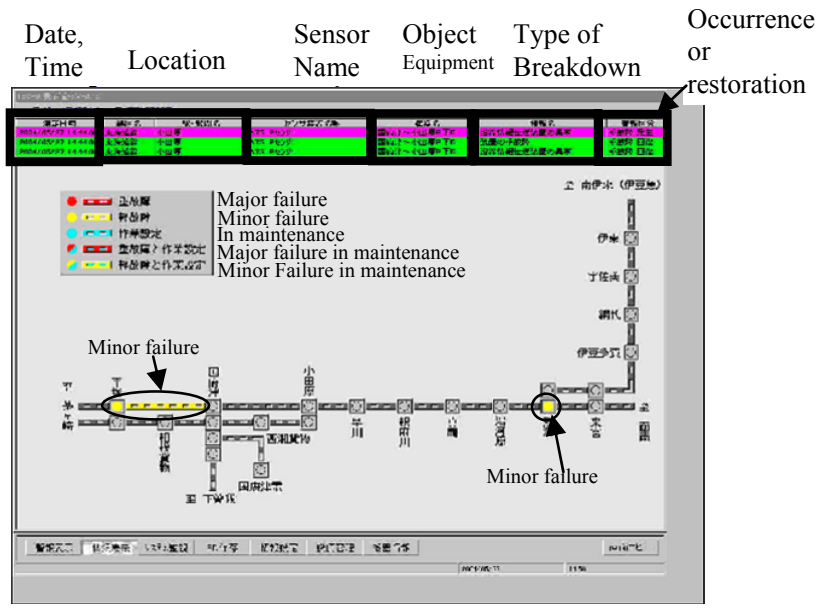

Figure 3: $\quad$ Example of the route map.

it is an occurrence or a restoration. When a breakdown or a recovery occurs, the display control also gives a warning sound.

When a breakdown occurs somewhere, it is possible to show the location of the fault on a route map, where it can easily be seen and identified.

\section{Summary of locking error detector}

In Japan, point machines have an internal locking mechanism. With this indirect lock system, the tongue rails are locked into position by the lock rod through its connection with the connect rod and the front rod. 


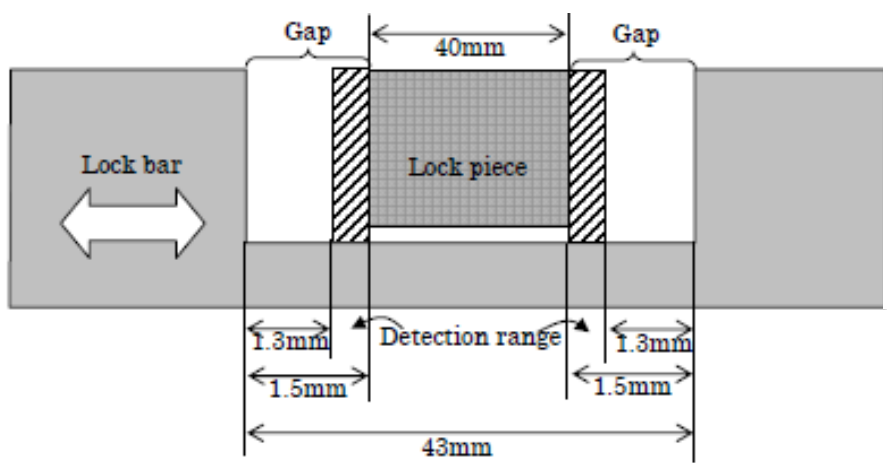

Figure 4: Detection range.

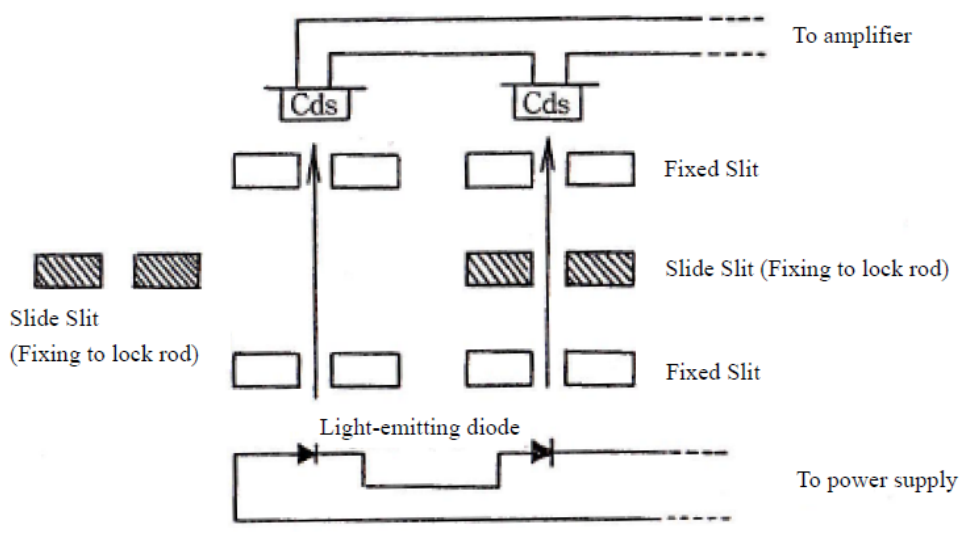

Figure 5: $\quad$ Structure of the locking error detector.

A locking error detector is provided to detect displacement of the mechanism before it leads to point failure. The locking error detector is installed in the point machine, and detects displacement from the change in the LED light, which passes through the slit. If this displacement becomes more than $1.3 \mathrm{~mm}$, an error alarm is sent to the control and display unit of the existing monitoring system.

\section{Summary of ES-type point machine monitoring}

\subsection{Constitution of the monitoring device of an ES-type point machine}

A breakdown of a point machine has a major impact on train operation. Therefore, it is important to monitor not only locking errors but also various other information.

The ES-type point machine that was introduced in 2002 uses a servo-motor.

With that change, we were able to monitor various kinds of information such as voltage, switching time, stroke, and torque. That information is sent to the 
control and display unit of the existing monitoring system by the monitoring device of the ES-type point machine. The constitution of the monitoring device of the ES-type point machine is shown in Figure 6.

\subsection{The recording function for items monitored}

Monitored items are as shown in Table 1.

The monitoring device can record the switching data a maximum of 10,000 times in every switching machine. An example of the switching data is shown in figure 7 . Torque value is indicated in brown. The limiting value is set lower than

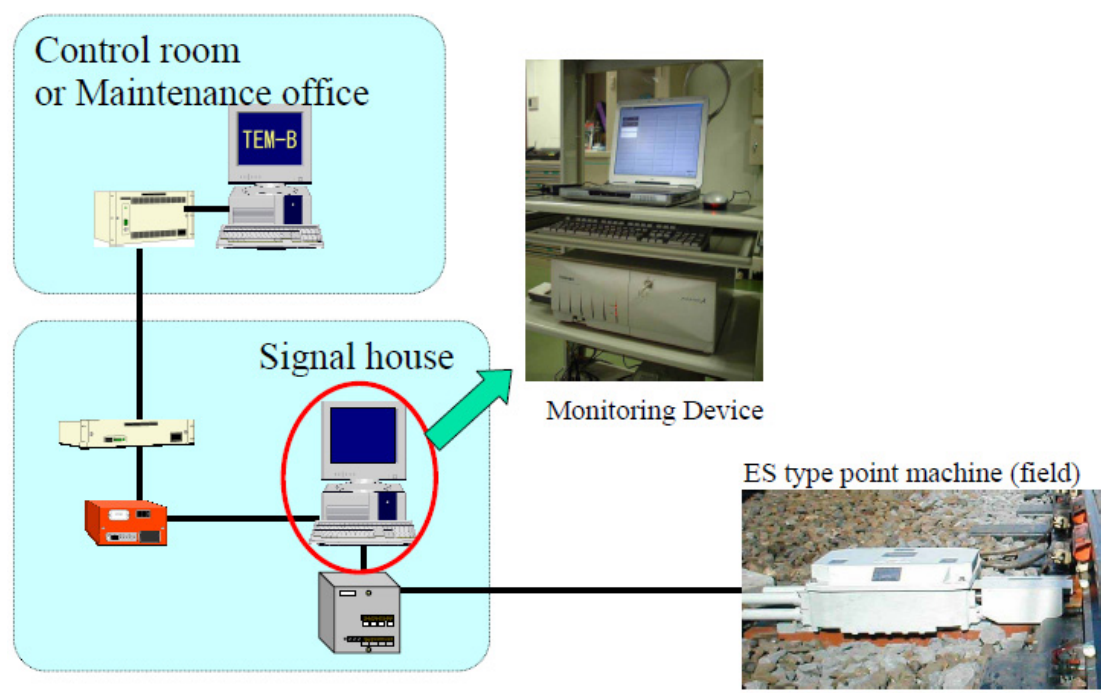

Figure 6: Constitution of the monitoring device of the ES-type point machine.

Table 1: Items monitored by the monitoring device of the ES-type point machine.

Every time of occurrence $\left\{\begin{array}{|l|l|}\hline \text { Supervisory item } & \text { Breakdown and Record contents } \\\right.$\cline { 2 - 3 } \text {$voltage } & \text { Voltage rise, voltage drop } \\ \hline \text { Control board abnormality } & \begin{array}{l}\text { One system major failure, One system minor failure } \\ \text { Control board Transmission abnormality }\end{array} \\ \hline \begin{array}{l}\text { Monitor repeater } \\ \text { abormality }\end{array} & \text { Monitor repeater transmission abnormality } \\ \text { Lock state } & \text { Limiting value setting possibility }(3.0 \mathrm{~mm}) \\ \hline \text { Switching force ,Torque } & \text { Limiting value setting possibility }(0 \sim 150 \%) \\ \hline \text { Switching time } & 0 \sim 6 \mathrm{sec} \\ \hline \text { Total number of switching } & \text { Up to } 400,000 \text { time } \\ \hline \text { Stroke } & 0 \sim+222 \mathrm{~mm} \\ \hline\end{array}$


the breakdown level. When it exceeds limiting value, information about the abnormality is sent to the control room and maintenance office. In addition, by accumulating data from many switching operations, when a breakdown occurs, we can find the cause quickly by comparing data from the abnormal situation with this accumulation of data from normal operation.

\subsection{Indication of trends}

Trend indications can be displayed, based on switching data retained. Trend in torque is illustrated in Figure 8. The maximum, mean, and minimum values of

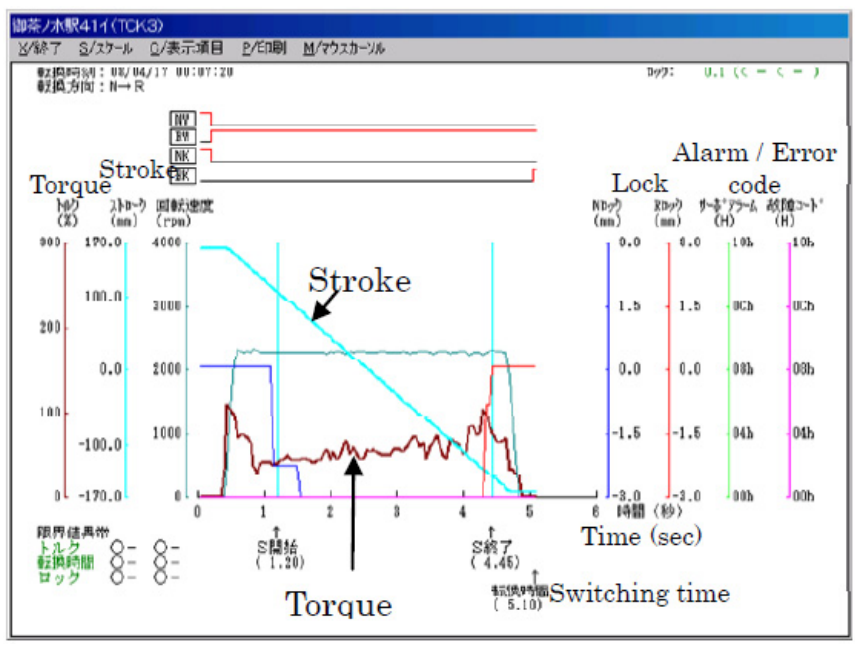

Figure 7: $\quad$ Example of switching data.

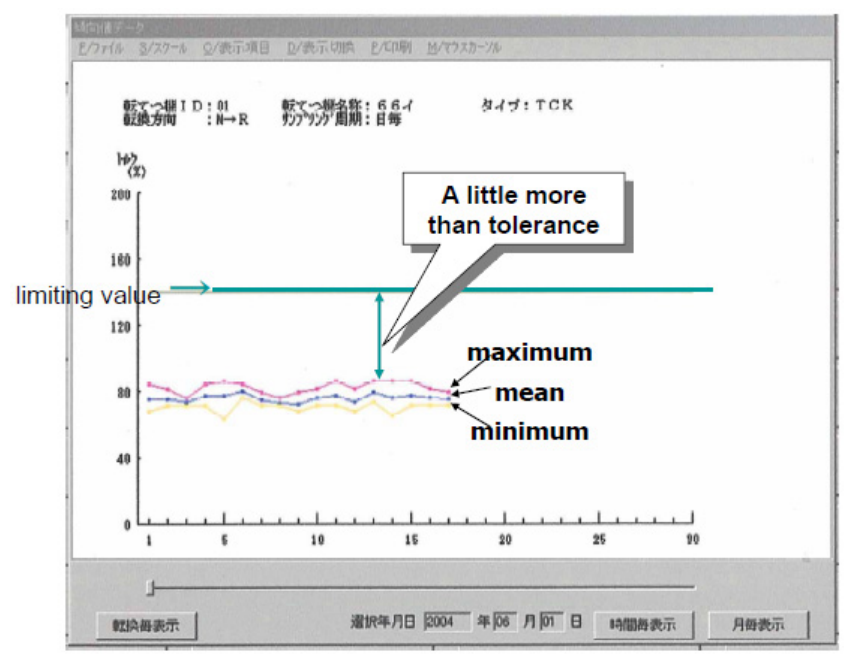

Figure 8: $\quad$ Indication of trends in torque. 
torque in one day are drawn in this graph. Changes in torque can be verified from this graph.

By checking the difference between the trend values and the limiting value, it is possible to judge whether it is necessary to go to the field location and make adjustments to the point machine. Trends in lock deviation can also be displayed. Trends can be indicated for every switching, every day, and every month.

\subsection{Function that retains a history of breakdowns}

The monitoring device can record history of breakdowns. When a breakdown occurs and when a recovery is made, date, time and switching machine name, breakdown type and occurrence of recovery are recorded. An example of the history of breakdown is shown in figure 9.

\section{Changes incorporated in the ES2-type point machine}

More than 200 ES-type point machines were introduced beginning in 2002, with the result that other data could be monitored, along with lighter weight of the equipment. After introduction, the ES-type point machine was improved in both hardware and software. However, there were problems in resistance to lightning and water, and there were limits on the required environmental conditions; for example, it could not be used in cold areas or in AC-electrified sections. Then we developed the ES2-type point machine that uses the concept of the ES type but also improves its features.

The development concept of the ES2-type point machine was as follows.

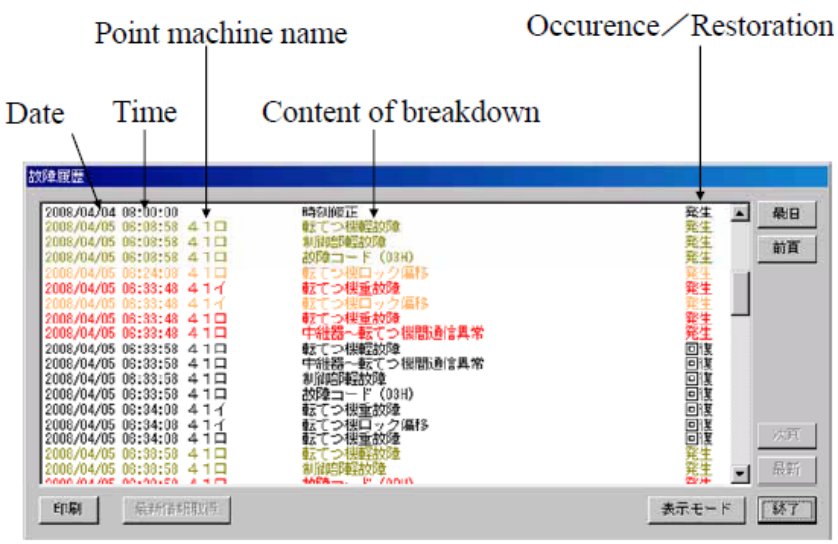

Figure 9: $\quad$ Example of the history of breakdowns. 


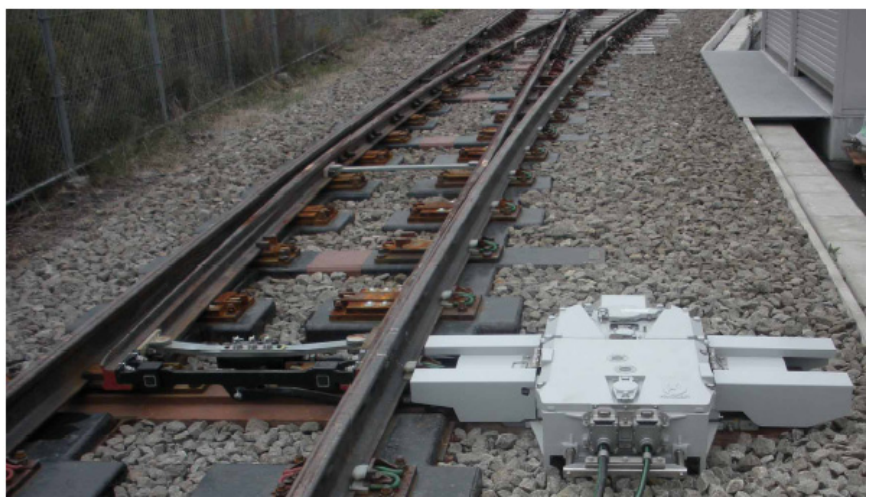

Figure 10: $\quad$ ES2-type point machine (new).
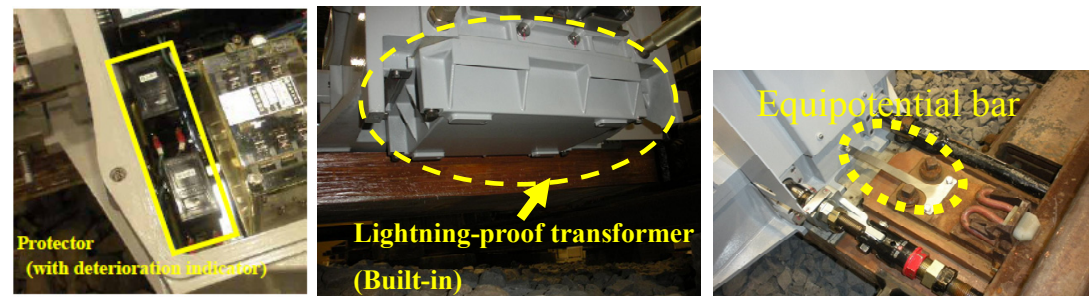

Figure 11: Improvement of lightning surge resistance.

\subsection{Assuring safe and stable operation}

a) Ability to ensure that there is no current in the circuit when the motor power source is turned off.

b) Built-in lightning-proof transformer and built-in safety device. Improvement of lightning surge resistance (ES type: $9 \mathrm{kV}->$ ES II type: $30 \mathrm{kV})$.

c) Improvement of water-tightness (ES type: Spray water -> ES2-type watertightness) were achieved.

d) Adopting a switching and lock mechanism that is resistant against breakdowns.

e) Opening direction indicator output at time of control failure. Adoption of point control relay and circuit control device.

f) Torque securing tolerance for turnout displacement.

\subsection{Simplification of installation and maintenance management}

a) Reduction of spare parts by permitting interchangeability between left-side and right-side uses.

b) Elimination of wiring work inside the switch.

c) Monitor unit that can be shared with ES type. 


\subsection{Designs for longevity and environmental considerations}

a) Make covers that can open and close and make it possible to replace each part individually (see figure 12). Reduce downtime and cut cost.

b) Ability to do overhauling at the manufacturers' workplace.

\subsection{Expansion of area where it is used}

a) Operating temperature $-20 \sim+60 \mathrm{deg} \mathrm{C}$.

b) Reduce switching time (6 seconds $->3.5$ seconds).

c) Motor power supply from $200 \mathrm{~V}+-10 \%$ to $105 \mathrm{~V}+-20 \%$.

d) Can be used in cant sections and AC-electrified sections.

\subsection{Revised characteristics of the monitoring device}

The revised characteristics of the monitoring device are as follows.

a) Records the turning angle of the switching roller in ES2 type so that the position of the switch rod and lock rod will be known.

b) Monitoring device can be used by both ES2 type and ES type.

c) When a switching order is given but the points do not switch, the switching data are recorded.

An example of the switching data is shown in figure 13.

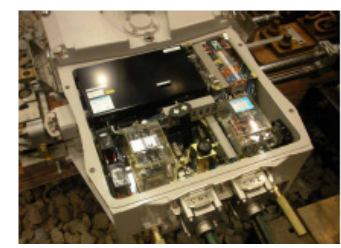

Figure 12: $\quad$ Point machine with the cover open.

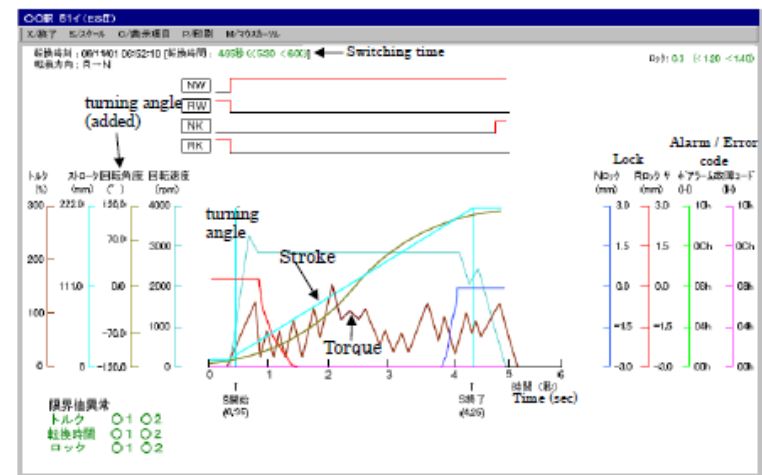

Figure 13: Example of the switching data (applies to both ES2 type and ES type). 


\section{Future research topics}

The ES-type point machine, for which a servo-motor was adopted, made possible the supervision of various items, recording of data and analysis of breakdowns. However, a switch consists of both the turnout and the switching machine, and the cause of failure to change can be the turnout itself. The turnout is inspected periodically, but is not monitored at all times. Torque values recorded with the monitoring device have been utilized to investigate the cause of breakdowns.

In the future, trouble with the turnout will also be monitored, using techniques now being researched. In addition, we will do research on how to know the signs of trouble before there is a breakdown.

\section{Conclusion}

When the ES-type point machine with a servo-motor was introduced in 2002, we could monitor not only the lock, but also the torque, stroke, voltage and other information. The next step was development of the ES2-type point machine, an improved ES type with greater stability. In the ES2-type point machine, monitoring device could be used by both ES2 type and ES type. Furthermore, it could record the turning angle of the switching roller in ES2 type.

In the future, trouble with the turnout mechanism will also be monitored through techniques now under research. We will also research how to know signs of trouble before there is a breakdown.

\section{References}

[1] Gregor, Treeg, and Sergej, Vlasenko, Railway Signalling \& Interlocking, pp.165-166, 2009.

[2] Kazue, Yasuoka, et al., "Practical application of the developed new pointmachine", International Symposium on Speed-up and Service Technology for Railway and Maglev Systems (STECH'06), 2006.

[3] JR Higashinihon Shingosetubi (jou) [JR East Signal Equipment (Upper Volume)], JR East General Education Center Electric Group, pp.10-25 - 1040, 2001. (in Japanese)

[4] Tentetsu-souchi [switch-and-lock movement apparatus], Japan Railway Electrical Engineering Association, pp.62-66, 1998. (in Japanese) 